\title{
Análise funcional dos sintomas depressivos do transtorno depressivo maior
}

\author{
Análisis funcional de los síntomas depresivos del trastorno depresivo mayor
}

\section{Depressive symptoms' functional analysis in major depressive disorder}

\author{
Ana Paula Gazzoni \\ Passo Fundo - RS/Brasil \\ ORCID: 0000-0002-3237-4117 \\ E-mail: anapaulagazzoni@gmail.com
}

\author{
Vinícius Renato Thomé Ferreira \\ IMED, Passo Fundo - RS/Brasil \\ ORCID: 0000-0001-9786-7775 \\ E-mail: vthome2@gmail.com
}

\begin{abstract}
Resumo
O Transtorno Depressivo Maior (TDM) causa prejuízos em todas as áreas na vida do indivíduo, e de acordo com a Organização Mundial da Saúde, será a doença mais incapacitante até o ano de 2020. Objetivou-se propor um entendimento sobre os sintomas depressivos do TDM presentes no DSM-5 pela perspectiva da análise do comportamento (AC). Os sintomas depressivos do TDM ocorrem principalmente devido à estimulação aversiva, por reforço negativo (comportamentos de fuga e esquiva), por processos de extinção e por baixo reforçamento positivo de comportamentos não depressivos. A análise funcional da relação aprendida entre o organismo e o ambiente é necessária para avaliar os sintomas depressivos, que resultam desta interação. Fatores relacionais e culturais, como interações familiares e sociais, o sexo/gênero, comportamento verbal e práticas culturais também precisam ser considerados na análise dos sintomas depressivos do TDM para se compreender sua variabilidade.
\end{abstract}

Palavras-chaves: Transtorno depressivo maior; Depressão; Comportamentalismo.

\section{Resumen}

El trastorno depresivo mayor (TDM) causa daños en todas las áreas de la vida de una persona y, según la Organización Mundial de la Salud, será la enfermedad más incapacitante para el año 2020. El objetivo fue proponer una comprensión de los síntomas depresivos del TDM presentes en el DSM-5 desde la perspectiva del análisis conductual (AC). Los síntomas depresivos de TDM ocurren principalmente debido a la estimulación aversiva, refuerzo negativo (conductas de escape y evitación), procesos de extinción y bajo refuerzo positivo de conductas no depresivas. Se requiere un análisis funcional de la relación aprendida entre el organismo y el ambiente para evaluar los síntomas depresivos que resultan de esta interacción. Los factores relacionales y culturales como las interacciones familiares y sociales, el sexo/género, el comportamiento verbal e practicas culturales también deben considerarse en el análisis de los síntomas depresivos de TDM para comprender su variabilidad.

Palabras clave: Trastorno depresivo mayor; Depresión; Conductualismo.

\begin{abstract}
Major Depressive Disorder (MDD) causes impairment in all areas of the individual's life, and it will be a more disabling disease by the year 2020 according to the World Health Organization. The objective was to propose an understanding of the depressive symptoms of MDD present in DSM-5 from the perspective of behavior analysis (BA). The depressive symptoms of MDD were mainly due to aversive stimulation, negative reinforcement (avoidance a fugue behaviors), extinction and low positive reinforcement of non-depressive behaviors. A functional analysis of the relationship between the organism and the environment is needed to evaluate depressive symptoms. Relational and cultural factors (e. g., family and social), sex/gender, verbal behavior, and cultural practices must be considered in order to understand variability of depressive symptoms in MDD.
\end{abstract}

Keywords: Depressive disorder, Major; Depression; Behaviorism. 
Os transtornos de humor são considerados problemas de saúde pública. Estima-se uma prevalência destes quadros em cerca de 300 milhões de pessoas, atingindo em torno de $5 \%$ da população mundial, e devem ocupar o segundo lugar entre as doenças na população mundial até o ano de 2020 (Fernandes, Falcone, \& Sardinha, 2012; Organização Mundial da Saúde [OMS], 2017). Dentre os países da América Latina, o Brasil apresenta maior prevalência de quadros depressivos, estimando-se que $5,8 \%$ da população seja afetada, totalizando 11,5 milhões de pessoas (OMS, 2017). Os transtornos de humor têm como principal característica prejuízos afetivos, atingindo o humor de maneira significativa. Constituem transtornos de humor o Transtorno Depressivo Maior (TDM), o transtorno disruptivo da desregulação do humor, o transtorno depressivo persistente (distimia), o transtorno disfórico pré-menstrual, o transtorno induzido por substâncias/medicamento, o transtorno depressivo devido a outra condição médica, outro transtorno depressivo especificado e transtorno depressivo não especificado (American Psychiatric Association [APA], 2014). A importância dos quadros depressivos está, principalmente, em comportamentos que apresentam redução da atividade (p. ex., apatia), sendo estes objetos de atenção clínica (Ferster, 1973).

O TDM é constituído pelos sintomas depressivos, que não são exclusivos deste quadro e podem estar presentes em outros diagnósticos, que são: humor deprimido e irritável, perda de prazer ou interesse em atividades, perda ou ganho de peso, insônia ou hipersonia, agitação ou retardo psicomotor, fadiga ou perda de energia, sentimento de inutilidade, culpa excessiva, dificuldade para pensar e concentrar, e pensamentos recorrentes de morte, gerando comprometimento no funcionamento do indivíduo e prejuízos na interação pessoal, social e profissional. A proposição de um diagnóstico de TDM para o DSM-5 exige pelo menos cinco sintomas dos critérios estabelecidos pelo período mínimo de duas semanas consecutivas, e que indiquem mudanças com relação ao seu funcionamento normal passado (prejuízo clinicamente significativo), e pelo menos um dos sintomas deve ser humor deprimido e irritável ou perda de interesse e do prazer (APA, 2014; Sadock, Sadock \& Ruiz, 2017). O TDM apresenta três vezes mais prevalência em indivíduos de 18 a 29 anos do que em indivíduos na terceira idade, e mulheres tem mais probabilidade de diagnóstico, com o surgimento dos primeiros sintomas na entrada da adolescência (APA, 2014). Desta forma, os prejuízos sociais dos sintomas depressivos são muito impactantes (Kupferberg, Bicks, \& Hasler, 2016).

Uma adequada compreensão sobre as causas dos sintomas depressivos, que originam o TDM, é tão importante quanto a avaliação da sua presença e intensidade, e as teorias psicológicas postulam diferentes explicações sobre o que origina e o que mantém estes sintomas. A proposta do artigo é oferecer uma possibilidade de entendimento funcional dos sintomas do TDM pela análise do comportamento (AC). Este estudo teórico traz contribuições da AC e oferece um entendimento funcional sobre os sintomas depressivos. Os materiais foram selecionados a partir da pertinência da discussão, a partir dos critérios diagnósticos do DSM-5 (APA, 2014) e da literatura da AC.

\section{Os Sintomas do TDM na Ótica da AC}

A AC, embasada nos estudos de Skinner, investiga o comportamento a partir de sua interação com seu ambiente (Carvalho Neto, 2002). O pressuposto central da AC é que não é possível entender o comportamento de forma isolada do ambiente, visto que é esta interação que aumenta ou diminui a probabilidade de sua emissão. A interação do organismo com o ambiente, então, acaba selecionando comportamentos, que ocorre em nível filogenético (em nível de espécie, pela seleção natural), ontogenético (o comportamento individual, construído e selecionado pelas histórias singulares de cada 
organismo) e cultural (na qual as interações entre o organismo e a cultura selecionam os comportamentos) (Skinner, 1974/1982).

O ambiente para a AC não está reduzido aos aspectos materiais do universo com o qual o organismo se relaciona, mas inclui a história de vida, as relações interpessoais e a sociedade como elementos que selecionam o comportamento. Pensamentos e sentimentos são considerados também como comportamentos porque também são selecionados pelo ambiente através da interação estabelecida (Moreira \& Medeiros, 2007), apenas com a diferença de que são privados, ou seja, existe um observador (a própria pessoa) quem os identifica e os experiencia (Skinner, 1953/2003). O aprendizado é um elemento central na AC, porque se a interação com o ambiente (físico e as relações sociais) é o que seleciona os comportamentos do organismo, pode-se dizer então que o comportamento, seja ele "sintomático" ou não, é aprendido (Skinner, 1953/2003). O entendimento de como ocorre este processo de aprendizado é fundamental para a AC, pois isso permite entender como o quadro foi se constituindo e também permite avaliar e construir as estratégias terapêuticas (Ferster, 1973).

$\mathrm{O}$ primeiro sintoma apresentado com relevância clínica no TDM pelo DSM-5 é o humor deprimido ou irritável (APA, 2014). Enquanto a tristeza faz parte de um repertório funcional de comportamentos, em nível patológico o humor deprimido se caracteriza como uma tristeza intensa, duradoura e que interfere de maneira clinicamente significativa em outros comportamentos (Sadock, Sadock, \& Ruiz, 2017). O humor deprimido pode ser considerado uma expressão mais intensa da tristeza, que atinge níveis de elevado sofrimento, estando associado à ocorrência de frequente estimulação aversiva na história de condicionamento do organismo. A estimulação aversiva pelo ambiente pode ser indutora de estados emocionais de ansiedade, frustração, raiva e de tristeza no organismo (Skinner, 1953/2003; Catania, 1999). Ambientes e pessoas que emitem com frequência estimulação aversiva, que pode ocorrer na forma de críticas, violência, etc., podem se tornar estímulos diferenciais para eliciar estas respostas emocionais (Ferster, 1973). Se a estimulação aversiva for muito intensa ou frequente, poderá ser generalizada (Moreira \& Medeiros, 2007) para outros ambientes, pessoas ou relacionamentos, e as respostas emocionais de tristeza podem assim tornar-se frequentes e com relevância clínica.

A tristeza pode ser igualmente um comportamento operante. Por exemplo, se uma pessoa se mostra "triste" para outras, é possível que estes outros parem de falar ou fazer coisas que sejam desagradáveis (aversivas) para a pessoa que apresenta essa tristeza; desta forma, ocorre um reforço negativo, ou seja, há a retirada de algo aversivo como consequência da resposta, poupando-o de mais sofrimento; assim o se apresentar de forma triste ("estar triste") se caracterizaria como comportamento de esquiva ou de fuga (Skinner, 1953/2003). A tristeza pode também ser positivamente reforçada (Skinner, 1953/2003; Catania, 1999) quando após a emissão de comportamentos queixosos e tristes há manifestação de cuidado, carinho e atenção por familiares, amigos e outras pessoas importantes, que talvez não dariam este tipo de reforçamento de outra forma. $\mathrm{O}$ reforço negativo consiste na eliminação ou evitação de algo aversivo como consequência do comportamento, enquanto o reforço positivo é o acréscimo de algo como consequência do comportamento (Skinner, 1953/2003; Moreira \& Medeiros, 2007). Finalmente, a perda de reforçadores, que podem se caracterizar como situações ou pessoas que são reforçadores positivos (p. ex., demissão, mudança de cidade), sendo caracterizada como punição negativa, também pode provocar tristeza (Rico, Golfeto, \& Hamasaki, 2012; Catania, 1999). Se esta perda de reforços perdurar, ocorrerá extinção (Skinner, 1953/2003; Catania, 1999) dos comportamentos e consequente redução do repertório.

Em relação ao segundo sintoma do TDM, que é a perda de interesse nas atividades que antes geravam prazer, este pode ocorrer por 
meio de extinção ou por reforçamento negativo. No caso da extinção, um ambiente com baixa frequência de reforçamento pode fazer com que o organismo tenha "desinteresse" em se comportar, como por exemplo, participar de interações sociais (Skinner, 1953/2003; Catania, 1999). Interações sociais que não resultam em elogios nem incentivos com frequência, ou ambientes que não tenham ou ofereçam poucos reforçadores naturais podem gerar extinção de comportamentos. O reforçamento negativo também pode aumentar a frequência de comportamentos de perda de interesse, pois se o ambiente emitir muita estimulação aversiva como consequência de comportamentos do organismo, a esquiva ou a fuga por meio de desinteresse (p. ex., "não fazer" ou "não dizer nada" para evitar uma crítica) é uma estratégia que pode reduzir esse sofrimento (Skinner, 1953/2003; Catania, 1999; Rico, Golfeto, \& Hamasaki, 2012).

O terceiro sintoma, que consiste em alterações de peso, pode estar presente no TDM na forma de aumento ou perda de peso corporal na taxa de 5\% em um mês (APA, 2014). O ganho de peso pode estar associado como um aumento do comportamento de comer como forma de aliviar estímulos emocionais aversivos (reforço negativo) e por meio do prazer que a comida proporciona (reforçamento positivo). Se o organismo está sob controle aversivo, as reações emocionais como ansiedade e tristeza podem ser negativamente reforçadas pela ingestão de alimentos apetitivos, aliviando (comportamento de fuga) a tristeza presente no TDM. A perda de peso, por sua vez, pode ser resultado de comportamentos de fuga e esquiva. $\mathrm{O}$ ato de se alimentar está usualmente associado a interações sociais (por exemplo, fazer uma refeição à mesa com outras pessoas), e a recusa a comer pode ser uma fuga ou esquiva de interações sociais aversivas (Ferster, 1973), o que acaba por reduz o repertório comportamental do organismo (Skinner, 1953/2003; Catania, 1999). Eventualmente pode ocorrer reforço positivo pela perda de peso corporal, considerando-se que há padrões sociais que privilegiam a magreza (Moriyama
\& Amaral, 2007). O reforço positivo ocorre quando se escuta que "Pelo menos você está mais magra/o". Além disso, a redução do comportamento de comer pode ser uma consequência da generalização do processo de extinção de outras respostas do organismo pela redução da efetividade de reforçadores (apatia) (Ferster, 1973), sendo resultado de uma baixa frequência de reforçamento do organismo pelo ambiente.

O aumento ou a redução clinicamente significativa do sono no TDM podem ocorrer no âmbito geral da extinção de comportamentos, como apresentado na redução de apetite (Ferster, 1973). A ocorrência frequente de eventos privados (pensamentos e sentimentos) associados ao sentimento de culpa (Rico, Golfeto, \& Hamasaki, 2012) podem dificultar que o organismo relaxe e possa iniciar o processo de indução do sono (Morin, Bootzin, Buysse, Edinger, Espie, \& Lichstein, 2006; Kaplan \& Harvey, 2016). Já o aumento do sono pode ser devido a comportamentos de esquiva ou fuga do contexto aversivo associado ao ambiente (Skinner, 1953/2003; Catania, 1999; Moreira \& Medeiros, 2007): se manter-se consciente mantém o organismo em contato com a estimulação aversiva, então o dormir se torna comportamento de fuga (elimina interações aversivas com outras pessoas) ou de esquiva (evita interagir com outras pessoas), sendo, portanto, negativamente reforçado. É também possível que o aumento da resposta de dormir seja um dos fenótipos da apatia.

O retardo ou a agitação psicomotora podem estar relacionadas também a um ambiente pouco reforçador ou à estimulação aversiva. A redução das atividades (retardo psicomotor) pode ser o resultado de extinção quando não ocorrem reforçamento dos comportamentos do organismo (Skinner, 1953/2003), gerando apatia (Ferster, 1973). Pode acontecer também que a ocorrência de eventos aversivos cause redução de comportamentos; experimentos de punição por meio de intervalo variável (IV) ou intervalo fixo (IF) sem possibilidade de comportamentos de esquiva ou fuga ocasionam a redução do repertório geral de respostas do organismo 
(Catania, 1999). Quando o/a cliente reduz seu repertório comportamental pode também estar menos vulnerável à estimulação aversiva do ambiente, constituindo-se desta forma em comportamento de esquiva ou fuga. Quanto à agitação, estudos em laboratório indicaram que a estimulação aversiva pode provocar comportamentos de agitação, que em termos humanos poderíamos chamar de frustração ou raiva (Catania, 1999). Agitação também pode ser utilizado como comportamento de fuga de situações associadas à estimulação aversiva condicionada geradoras de ansiedade (p. ex., silêncios ou inatividade): nestes casos, qualquer comportamento, incluindo a agitação improdutiva, pode ser tomado como alternativa para evitar a situação aversiva (Ferster, 1973) que ocorreria se o organismo "não fizesse nada" ou "ficasse quieto".

A fadiga ou baixa energia podem ocorrer como o resultado da pouca estimulação do ambiente, também como resultado de processos de extinção (Ferster, 1973; Catania, 1999), pela redução de reforçamento de comportamentos ativos (caminhar, trabalhar, realizar interações sociais, etc.). Por outro lado, a punição de comportamentos que o organismo venha a emitir (p. ex., conversar, trabalhar) pode aumentar a frequência da fadiga: se o organismo faz alguma coisa e é punido por isso, a fadiga visa ser uma resposta de "proteção" para evitar punições futuras (Moreira \& Medeiros, 2007).

Os sentimentos de inutilidade podem ser resultado de uma avaliação do próprio comportamento quando comparado às respostas das demais pessoas, ou em comparação com o comportamento apresentado no passado. Quando o/a cliente observa que não consegue mais emitir resposta devido aos demais sintomas do TDM (p. ex., trabalhar, sair, etc.), pode-se estabelecer uma autorregra de que é ou está "inútil", que é confirmada pelo aumento de críticas das pessoas próximas ao/à cliente quando referem que "não é mais o/a mesmo/a" de antes. Sentimentos de inutilidade podem estar associados a uma história de críticas e/ou de cobranças excessivas do ambiente por um determinado desempenho (Rico, Golfeto, \& Hamasaki, 2012). A presença dessas expectativas e cobranças pode criar uma autorregra de que ele/a deve estar sempre no máximo de seu desempenho, tendo que alcançar sempre o sucesso e atender às expectativas das pessoas próximas. Quando isso não ocorre, podem surgir eventos privados na forma de autocobranças e a conclusão de que o insucesso é consequência de uma inutilidade ou incapacidade para realizar as tarefas.

Embora seja necessário definir operacionalmente o que é a culpa, pode ser considerada como um sentimento aversivo como resultado das críticas do ambiente. $\mathrm{O}$ sentimento de culpa pode ter sua origem na percepção de inutilidade e de ter comportamentos que são reprovados (punidos) pelo ambiente social (Rico, Golfeto, \& Hamasaki, 2012), de forma semelhante com o que ocorre com a sensação de inutilidade. A ocorrência de condicionamento por meio de punições frequentes pode despertar sentimentos de culpa, criar uma regra rígida de desempenho e eliciar sentimentos aversivos fortes quando esta regra rígida não for cumprida. A rigidez da regra pode ocorrer por um alto temor da punição, porque se no passado pequenos desvios de regra foram severamente punidos, estima-se que no presente haverá grande preocupação em não as violar.

A dificuldade de concentração ou de pensar (Bandini \& Delage, 2012) pode também ser resultado da estimulação aversiva, fazendo com que também os eventos privados tenham sua frequência reduzida. A ocorrência de punição possui como efeito a redução do repertório comportamental (Banaco, 2004). Dificuldades de pensar, se concentrar e resolver problemas são eventos privados também passíveis de controle pelo ambiente, e podem ser reduzidos num contexto de estimulação aversiva. É possível também que a dificuldade de concentração seja utilizada como reforçador negativo, evitando com que a pessoa possa estar em contato com ambientes, estímulos ou situações que estejam sendo aversivas (p. ex., no contexto do trabalho). 
Finalmente, sobre a ideação suicida, pode-se considerar que a ocorrência deste comportamento evite ou elimine estimulação aversiva: a possibilidade de terminar com a própria vida pode ser um comportamento extremo de fuga (se for considerando um ambiente aversivo já presente) ou de esquiva (como meio de evitar novas punições futuras e mais sofrimento) (Cavalcante, 1997). A ideação suicida pode ser entendida como a presença de um evento privado de autocontrole extremo, no qual o organismo contempla uma restrição física total como saída para a eliminação de estimulações aversivas (Skinner, 1953/2003). A tabela 1 consiste numa proposta de síntese dos principais processos comportamentais associados aos sintomas depressivos descritos acima.

Tabela 1.

Funções que podem estar presentes nos sintomas do TDM

\begin{tabular}{|c|c|c|c|c|c|}
\hline \multirow{3}{*}{ Sintoma } & \multirow{3}{*}{$\begin{array}{l}\text { Estimulação } \\
\text { aversiva }\end{array}$} & \multicolumn{4}{|c|}{ Condicionamento operante } \\
\hline & & \multirow{2}{*}{$\mathrm{R}+$} & \multicolumn{2}{|c|}{ R- } & \multirow{2}{*}{ Extinção } \\
\hline & & & Fuga & Esquiva & \\
\hline Humor deprimido & $\mathrm{X}$ & $\mathrm{X}$ & & $\mathrm{X}$ & $\mathrm{X}$ \\
\hline Perda do interesse e prazer & $X$ & & $X$ & $X$ & $X$ \\
\hline Aumento de peso & $\mathrm{X}$ & & $X$ & & \\
\hline Diminuição de peso & $\mathrm{X}$ & $X$ & $\mathrm{X}$ & $\mathrm{X}$ & $\mathrm{X}$ \\
\hline Redução de sono & $\mathrm{X}$ & & & & \\
\hline Aumento de sono & $\mathrm{X}$ & & $\mathrm{X}$ & $\mathrm{X}$ & \\
\hline Retardo psicomotor & $\mathrm{X}$ & & $\mathrm{X}$ & $X$ & $\mathrm{X}$ \\
\hline Agitação psicomotora & $\mathrm{X}$ & & $\mathrm{X}$ & $X$ & \\
\hline Fadiga & $\mathrm{X}$ & & $\mathrm{X}$ & $\mathrm{X}$ & $\mathrm{X}$ \\
\hline Sentimento de inutilidade & $\mathrm{X}$ & & & & \\
\hline Sentimento de culpa & $\mathrm{X}$ & & & & \\
\hline Dificuldade de concentração e decisão & $\mathrm{X}$ & & $\mathrm{X}$ & $X$ & \\
\hline Ideação suicida & $\mathrm{X}$ & & $\mathrm{X}$ & $\mathrm{X}$ & \\
\hline
\end{tabular}

Nota. Tabela elaborada pelos autores

\section{Discussão}

Não há uma causa única para os sintomas depressivos, visto que são vários os processos implicados no seu surgimento (Ferster, 1973). A ocorrência frequente de estimulação aversiva, de reforçamento negativo e de processos de extinção, que pode ser observada na Tabela 1, aponta que estas funções estão muito presentes nos sintomas do TDM. A estimulação aversiva parece ser a principal fonte de sintomas depressivos, principalmente porque sua presença elicia respostas emocionais que definimos como tristeza, raiva e frustração (Catania, 1999). A punição apresenta vários efeitos indesejáveis pela eliciação de respostas emocionais que, ao mesmo tempo em que podem ser considerados sintomas depressivos, podem contribuir com a ocorrência de comportamentos de fuga $\mathrm{e}$ esquiva associados ao TDM. Portanto, a estimulação aversiva possui um papel fundamental na origem e na manutenção do TDM.

O reforçamento negativo está diretamente relacionado à estimulação aversiva. Os comportamentos de fuga e esquiva ocorrem na presença desta estimulação, seja para eliminar a que está ocorrendo (comportamento de fuga), seja para evitar sua ocorrência futura (comportamento de esquiva) a partir da apresentação do estímulo discriminado (Skinner, 1953/2003). Contudo, mesmo sendo bem sucedida momentaneamente no controle dos estímulos aversivos, a ocorrência frequente de reforço negativo, se generalizado, pode trazer prejuízos em outras áreas. Quando isto ocorre, os sintomas depressivos podem ser fonte de atenção clínica, 
pois a utilização constante de reforçamento negativo pelo organismo pode dificultar ao organismo o acesso a reforçadores positivos de outros comportamentos (Skinner, 1953/2003; Ferster, 1973; Catania, 1999).

Os processos de extinção também estão associados à ocorrência de sintomas depressivos, principalmente os considerados "negativos" (Sadock, Sadock, \& Ruiz, 2017), ou seja, aqueles que implicam na redução da expressão e frequência de comportamentos. $\mathrm{O}$ TDM apresenta prejuízos tanto pela ocorrência dos sintomas positivos (agitação, ideação suicida, tristeza) tanto quanto por negativos (perda de sono, de apetite e fadiga), e esta redução de comportamentos acontece tanto pela ocorrência de punição quando pela ocorrência da extinção. No caso dos sintomas positivos, a punição pode reduzir comportamentos adaptados (funcionais, não depressivos) pela ocorrência da estimulação aversiva (Skinner, 1953/2003); no caso dos sintomas negativos, a extinção pode ocorrer pela ausência de reforçamento positivo de comportamentos, reduzindo respostas a variabilidade de respostas (Catania, 1999; Skinner, 1953/2003). Observa-se, portanto, que estes três mecanismos podem ocorrer de maneira conjunta no estabelecimento do TDM.

É também notável a baixa ocorrência de reforçamento positivo nos sintomas do TDM. A retirada de reforços positivos em indivíduos que possuem deficiências em seus repertórios comportamentais pode se tornar um fator de risco para sintomas depressivos (Reisinger, 1972; Ferster, 1973; Abreu, 2006; Azevedo, Almeida, \& Moreira, 2009). Situações como a retirada de reforçadores positivos (por punição negativa ou extinção) como consequência de mudanças ambientais (perda de pessoas importantes, mudanças de cidade, desemprego, etc.) podem ser fatores de risco, considerandose o contexto geral de reforçadores (Ferster, 1973; Abreu, 2006). A ocorrência de reforçamento positivo favorece a variabilidade de comportamento e aumentam a frequência de respostas emocionais consideradas "prazerosas", o que poderia gerar um efeito protetivo aos sintomas depressivos (Ferster,
1973; Hersen, Eisler, Alford, \& Agras, 1973; Hünziker, 2001; Abreu, 2006). Nota-se também que pode ocorrer reforçamento positivo de sintomas depressivos quando o ambiente identifica estes sintomas nos pacientes e a partir daí despende atenção, carinho e cuidado a eles que de outra forma não ocorreriam.

Se por um lado o ambiente pode estar emitindo estimulação aversiva, também devese considerar que a relação que o organismo tem com o ambiente mantém estes sintomas. Dessa forma, é importante identificar o repertório comportamental e considerar a relação funcional entre o comportamento e o ambiente, bem como investigar as variáveis que antecedem e que sucedem um determinado comportamento (Cavalcante, 1997; Martin \& Pear, 2009). Investigar a história de condicionamento permite a compreensão precisa e, para a identificação do que realmente pode ter ocorrido. O comportamento é função da relação estabelecida entre o organismo e o ambiente (Skinner, 1953/2003) e não é um mero resultado passivo desta estimulação. Da mesma forma que o ambiente consequência (reforça, pune) ou não (extingue) comportamentos, o organismo também o modifica por meio de suas respostas (Skinner, 1953/2003). Pode ser mais útil avaliar os sintomas depressivos num contexto de metacontingências (Glenn, 1988), onde, ao mesmo tempo em que o cliente recebe a estimulação ambiental (estímulo, consequência) também emite respostas que funcionam como estímulos discriminativos para as outras pessoas do ambiente social. Portanto, esta interação sempre deve ser considerada quando se avaliam e tratam os transtornos, e é o principal foco de trabalho na clínica analítico-comportamental. As combinações e apresentações dos sintomas do TDM são únicas, porque cada história de condicionamento é única.

O papel das respostas emocionais é muito importante no TDM, tanto que é considerado um dos transtornos chamados de "afetivos". Ao mesmo tempo em que as emoções de raiva e tristeza se constituem em 
sintomas centrais no TDM, eles também contribuem funcionalmente para a manutenção de outros comportamentos, principalmente de fuga e esquiva. Isso ocorre porque as respostas emocionais podem ser consideradas tanto respondentes quanto operantes, dependendo da situação (Darwich \& Tourinho, 2005). Essa complexa composição de comportamentos respondentes e operantes precisa ser levada em consideração na avaliação e no tratamento dos sintomas depressivos.

A proposição da hipótese diagnóstica de TDM levará em conta a avaliação clínica sobre a situação concreta (Sadock, Sadock, \& Ruiz, 2017). Não é necessária a presença de todos os sintomas depressivos para uma hipótese de TDM, visto que apenas o atendimento a cinco critérios diagnósticos, sendo um deles o humor deprimido ou a perda do interesse ou prazer na realização de atividades, por um tempo mínimo de duas semanas e que implique em prejuízo clinicamente significativo é suficiente (APA, 2014). Ainda, pode existir variabilidade nos próprios sintomas, que variam para mais ou para menos no mesmo (p. ex., sono e apetite), o que pode ser um desafio na avaliação clínica. Cada história individual irá selecionar as respostas a partir das funções mais presentes no ambiente, e por isso há diferentes fenótipos comportamentais: as funções destes comportamentos são diferentes de pessoa para pessoa.

Fatores culturais mais amplos também interferem nos sintomas depressivos, como por exemplo as questões de sexo e gênero (Correia \& Borlotti, 2011). Ser mulher aumenta o risco do desenvolvimento de sintomas depressivos, tendo em vista que há maior vulnerabilidade para ser vítima de violência, de ter menores ganhos financeiros em comparação com o homem, ter maiores cobranças de desempenho nas relações interpessoais e cuidados com a família (filhos, pais, etc.). Estes elementos precisam ser levados em conta na avaliação e no tratamento do TDM, pois são macrocontingências (Glenn, 2015) que afetam o comportamento, podendo ser ou não fatores protetivos. Portanto, a compreensão do comportamento verbal (Skinner, 1957/2014) e das práticas culturais são necessárias para a compreensão da estimulação aversiva, dos reforços negativos, da extinção e da baixa ocorrência de reforçamento positivo nos quadros de TDM.

\section{Considerações Finais}

Os sintomas depressivos presentes no TDM, conforme os critérios diagnósticos do DSM-5 ocorrem principalmente devido à grande frequência da estimulação aversiva, por reforço negativo (comportamentos de fuga e esquiva), extinção e por baixo reforçamento positivo de sintomas não depressivos, embora alguns sintomas depressivos possam também ser positivamente reforçados. Mesmo que o ambiente forneça estimulação aversiva, é fundamental considerar a relação aprendida entre o organismo e o ambiente para avaliar o comportamento, por meio do conhecimento da história única de condicionamento. Fatores relacionais e culturais, como as relações interpessoais (familiares, sociais), sexo, gênero e práticas culturais também precisam ser avaliados funcionalmente para se compreender a variabilidade dos sintomas depressivos.

A leitura externalista e relacional da $\mathrm{AC}$ sobre os sintomas depressivos pode oferecer uma compreensão diferenciada em relação a outras teorias psicológicas, de cunho internalista e mentalista. Com isso não se quer dizer que não existam componentes biológicos que estejam associados aos sintomas depressivos, como alterações em neurotransmissores, mas sim que a ênfase da análise deve recair sobre os aspectos relacionais entre o organismo e o ambiente. Essa perspectiva direciona para uma leitura que inclui a interação como elemento clínico central no TDM. A análise funcional dos casos concretos deve ser conduzida para identificar a ocorrência destes processos e a interação do organismo com o ambiente. O comportamento é função da interação entre o organismo e o ambiente, e sempre estas duas variáveis precisam ser consideradas em interação na análise dos sintomas depressivos pela clínica analítico-comportamental. 


\section{Referências}

Abreu, P. B. (2006). Terapia analíticocomportamental da depressão: uma antiga ou uma nova ciência aplicada? Revista de Psiquiatria Clínica, 33(6), 322-328. doi: 10.1590/s0101-60832006000600005

American Psychiatric Association [APA]. (2014). Manual Diagnóstico e Estatístico de Transtornos Mentais - DSM-5. Porto Alegre: Artmed.

Azevedo, L. A., Almeida, T. C., \& Moreira, A. H. (2009). "O resfriado da psiquiatria": a depressão sob o ponto de vista analíticocomportamental. Transformações em Psicologia, 2(1), 65-85. Recuperado de http://pepsic.bvsalud.org/scielo.php?script= sci_arttext\&pid=S2176106X2009000100005\&lng=pt

Banaco, R. A. (2004). Punição positiva. In: C. N. Abreu \& H. J. Guilhardi (Eds.), Terapia Comportamental e Cognitivocomportamental: práticas clínicas (pp. 6171). São Paulo: Roca.

Bandini, C. S. M., \& Delage, P. E. G. A. (2012). Pensamento e criatividade. In: Hubner, M. M. C., \& Moreira, M. B. (Eds.), Temas clássicos da psicologia sob a ótica da Análise do Comportamento (pp. 117-128). Rio de Janeiro: Guanabara Koogan.

Carvalho Neto, M. B. (2002). Análise do comportamento: behaviorismo radical, análise experimental do comportamento e análise aplicada do comportamento. Interação em Psicologia, 6(1), 13-18. doi: 10.5380/psi.v6i1.3188

Catania, A. C. (1999). Aprendizagem: Comportamento, Linguagem e Cognição. Porto Alegre: Artes Médicas Sul.

Cavalcante, S. N. (1997). Notas sobre o fenômeno depressão a partir de uma perspectiva analítico-comportamental. Psicologia: Ciência e Profissão, 17(2), 212. doi: $10.1590 / \mathrm{s} 1414-$ 98931997000200002

Correia, K. M. L., \& Borloti, E. (2011). Mulher e depressão: uma análise comportamental-contextual. Acta Comportamentalia: Revista Latina de
Análisis de Comportamiento, 19(3), 359373. Recuperado de http://pepsic.bvsalud.org/scielo.php?script= sci_arttext\&pid=S0188$81452011000300007 \& \operatorname{lng}=\mathrm{pt} \& \mathrm{t} \operatorname{lng}=\mathrm{pt}$

Darwich, R. A., \& Tourinho, E. Z. (2005).

Respostas emocionais à luz do modo causal de seleção por consequências. Revista Brasileira de Terapia Comportamental e Cognitiva, 7(1), 107-118. Doi:

10.31505/rbtcc.v7i1.46

Fernandes, C. S., Falcone, E. M. O., \& Sardinha, A. (2012). Deficiências em habilidades sociais na depressão: estudo comparativo. Psicologia: Teoria e Prática, 14(1), 183-196. Recuperado de http://pepsic.bvsalud.org/scielo.php?script= sci_arttext\&pid=S151636872012000100014\&lng=pt\&tlng=pt

Ferster, C. B. (1973). A functional analysis of depression. American Psychologist, 28(10), 857-870. doi: 10.1037/h0035605

Glenn, S. S. (1988). Contingencies and metacontingencies: Toward a synthesis of behavior analysis and cultural materialism. The Behavior Analyst, 11(2), 161-179. doi: https://doi.org/10.1007/bf03392470

Glenn, S. S. (2015). Comportamento individual, cultura e mudança social. Revista Brasileira de Análise do Comportamento, 11(2), 208-222. doi: 10.18542/rebac.v11i2.4015

Hersen, M., Eisler, R. M., Alford, G. S., \& Agras, W. S. (1973). Effects of token economy on neurotic depression: An experimental analysis. Behavior Therapy, 4(3), 392-397. doi: 10.1016/S00057894(73)80119-4

Hünziker, M. H. L. (2001). O desamparo aprendido e a análise funcional da depressão. In D. R. Zamignani (Eds.), Sobre Comportamento e Cognição: a aplicação da análise do comportamento $e$ da terapia cognitivo-comportamental no hospital geral e nos transtornos psiquiátricos (V. 3, pp. 145-153). Santo André: ESETec. 
Kaplan, K. A., \& Harvey, A. G. (2016). Tratamento dos transtornos do sono. In: Barlow, D. H. (Eds.), Manual clínico dos transtornos psicológicos: tratamento passo a passo (pp. 636-664). Porto Alegre: Artmed.

Kupferberg A, Bicks L, Hasler G. (2016). Social functioning in major depressive disorder. Neuroscience \& Biobehavioral Reviews (69), 313-332. doi: 10.1016/j.neubiorev.2016.07.002

Martin, G., \& Pear, J. (2009). Modificação de Comportamento: o que é e como fazer. São Paulo: Roca.

Moreira, M. B.; Medeiros, C. A. (2007). Princípios básicos de análise do comportamento. Porto Alegre: Artmed.

Morin, C. M., Bootzin, R. R., Buysse, D. J., Edinger, J. D., Espie, C. A., \& Lichstein, K. L. (2006). Psychological and behavioral treatment of insomnia: update of the recent evidence (1998-2004). Sleep, 29(11), 13981414. doi: $10.1093 /$ sleep/29.11.1398

Moriyama, J. D. S., \& Amaral, V. L. A. R. D. (2007). Transtorno dismórfico corporal sob a perspectiva da análise do comportamento. Revista Brasileira de
Terapia Comportamental e Cognitiva, 9(1), 11-25. doi: 10.31505/rbtcc.v9i1.143

Organização mundial de saúde [OMS]. (2017). Saúde mental: nova compreensão, nova esperança. Genova: OMS.

Reisinger, J. J. (1972). The treatment of "anxiety-depression" via positive reinforcement and response cost. Journal of Applied Behavior Analysis, 5(2), 125-130. doi: 10.1901/jaba.1972.5-125

Rico, V. V., Golfeto, R., \& Hamasaki, E. I. M. (2012). Sentimentos. In: Hubner, M. M. C., \& Moreira, M. B. (Eds.), Temas clássicos da psicologia sob a ótica da Análise do Comportamento (pp. 88-99). Rio de Janeiro: Guanabara Koogan.

Sadock, B. J., Sadock, V. A., \& Ruiz, P. (2017). Compêndio de Psiquiatria: Ciência do Comportamento e Psiquiatria Clínica (11 ed.). Porto Alegre: Artmed.

Skinner, B. F. (1957/2014). Verbal behavior. BF Skinner Foundation.

Skinner, B. F. (1974/1982). Sobre o behaviorismo. São Paulo: Cultrix.

Skinner, B. F. (1953/2003). Ciência e comportamento humano. São Paulo: Martins Fontes.

\section{Dados sobre os autores:}

- Ana Paula Gazzoni: Psicóloga clínica, especialista em Terapia Cognitivo-Comportamental pela IMED - Passo Fundo (RS). Formação em Terapia Cognitivo Comportamental na Infância e Adolescência pela ELO Psicologia e Desenvolvimento.

- Vinícius Renato Thomé Ferreira: Doutor em psicologia pela pela PUCRS (2008), graduado em psicologia pela Universidade de Passo Fundo (1998), especialista em Relações Familiares (UPF), e mestre em psicologia (PUCRS). Coordenou a estruturação da Escola de Psicologia da IMED e foi Diretor Acadêmico (2010-2012). Foi professor da UnC em Concórdia e Caçador, e da Universidade Regional Integrada, em Erechim e Santo Ângelo. Psicólogo clínico. Tem experiência na área de psicologia, com ênfase em avaliação terapêutica, especialmente sintomas depressivos, avaliação psicológica, psicopatologia e análise do comportamento. Professor e pesquisador do Programa de Pós-Graduação, mestrado, em Psicologia da IMED.

\footnotetext{
Declaração de Direito Autoral

A submissão de originais para este periódico implica na transferência, pelos autores, dos direitos de publicação impressa e digital. Os direitos autorais para os artigos publicados são do autor, com direitos do periódico sobre a primeira publicação. Os autores somente poderão utilizar os mesmos resultados em outras publicações indicando claramente este periódico como o meio da publicação original. Em virtude de sermos um periódico de acesso aberto, permite-se o uso gratuito dos artigos em aplicações educacionais e científicas desde que citada a fonte conforme a licença CC-BY da Creative Commons.
} 